\title{
Space weather in the EU's FP7 Space Theme
}

\section{Preface to the special issue on "EU-FP7 funded space weather projects"}

\author{
Paola Chiarini* $^{*}$ \\ Research Programme Officer, European Commission's Research Executive Agency, Space Research Unit, COV2, B-1049 Brussels, \\ Belgium \\ *Corresponding author: paola.chiarini@ec.europa.eu
}

The views and opinions expressed in this text are those of the author. They do not necessarily correspond to those of the organisation by which the person is employed.

Received 25 October 2013 / Accepted 28 October 2013

\begin{abstract}
Technological infrastructures in space and on ground provide services on which modern society and economies rely. Space weather related research is funded under the 7th Framework Programme for Research and Innovation (FP7) of the European Union in response to the need of protecting such critical infrastructures from the damage which could be caused by extreme space weather events. The calls for proposals published under the topic "Security of space assets from space weather events" of the FP7 Space Theme aimed to improve forecasts and predictions of disruptive space weather events as well as identify best practices to limit the impacts on space- and ground-based infrastructures and their data provision. Space weather related work was also funded under the topic "Exploitation of space science and exploration data", which aims to add value to space missions and Earth-based observations by contributing to the effective scientific exploitation of collected data. Since 2007 a total of 20 collaborative projects have been funded, covering a variety of physical phenomena associated with space weather, from ionospheric disturbances and scintillation, to geomagnetically induced currents at Earth's surface, to coronal mass ejections and solar energetic particles. This article provides an overview of the funded projects, touching upon some results and referring to specific websites for a more exhaustive description of the projects' outcomes.
\end{abstract}

Key words. EU funding - FP7 - space weather - EU research projects

\section{Why the EU funds space research?}

Under the Space Theme of the 7th Framework Programme for Research and Innovation (FP7), the European Union has been funding over EUR 37 million worth of space weather-related research through 20 collaborative projects involving approximately 165 organisations, including universities, research institutes and companies. The "research into reducing the vulnerability of space assets", from both space debris and space weather events, is an integral part of the European Space Policy.

The European Union's space strategy is conceived as a response to the social, economic and strategic challenges faced by society. Space activities, through scientific research and their direct applications, are acknowledged as strategic for their contribution to the construction of Europe and the competitiveness of the European Union. As the space sector contributes directly to the Europe 2020 Strategy $^{1}$, namely smart, sustainable and inclusive growth, the European Commission is tasked "to develop an effective space policy to provide the tools to address

\footnotetext{
1 "EUROPE 2020: A strategy for smart, sustainable and inclusive growth" COM (2010) 2020, http://ec.europa.eu/europe2020.
}

some of the key global challenges and in particular to deliver Galileo ${ }^{2}$ and GMES", 3,4

The Space Strategy Communication ${ }^{5}$ clearly recognises that the space infrastructure is critical for services on which contemporary society and economies rely. The modern technological infrastructures in space and on ground are at risk of damage or destruction by natural phenomena. Of increasing concern are extreme space weather events, which can have a serious impact and cause major failures, e.g., on board spacecraft, in electrical power grids, in telecommunications links (satellite, launcher and ground-based) and in navigation systems. The FP7 Work Programme 2010 for the Space Theme clearly stated that "during

\footnotetext{
${ }^{2}$ The Galileo programme is Europe's initiative for a state-of-the-art global satellite navigation system, providing a highly accurate, guaranteed global positioning service under civilian control. See http://www.gsa.europa.eu/galileo-0.

${ }^{3}$ Copernicus, previously known as GMES (Global Monitoring for Environment and Security), is the European Programme for the establishment of a European capacity for Earth Observation. See www.copernicus.eu.

${ }^{4}$ Communication "Towards a space strategy for the European Union that benefits its citizens", COM (2011) 152 final, 4 April 2011.

${ }^{5}$ Idem.
} 
the current approach of the next solar maximum (around 2012), more accurate prediction, assessment and early-warning capabilities of disruptive events that are to be expected as part of this cyclical phenomenon are particularly poignant". 6

The objective to protect the space- and ground-based infrastructures was translated into a specific research topic within the FP7 Space Theme.

\section{Space weather related calls for proposals in FP7}

Since 2007 six calls for proposals have been issued under the Space Theme. Space weather has been one of the topics of two calls under the area "Research into reducing the vulnerability of space assets".

In the call FP7-SPACE-2010-1, published in 2009, the topic "Security of space assets from space weather events" aimed to improve forecasts and predictions of disruptive space weather events as well as identify best practices to limit the impacts on space- and ground-based infrastructures and their data provision. Given the open approach of the topic, the research activities of the eight funded projects span to cover a variety of physical phenomena associated with space weather, from ionospheric disturbances and scintillation, to geomagnetically induced currents at Earth's surface, to coronal mass ejections and solar energetic particles (SEPs).

The call FP7-SPACE-2013-1, published in 2012, focused on the more accurate modelling and impact assessment of both the effects of the ionosphere on navigation signals, in particular on Global Navigation Satellite Systems (GNSS), and the interaction of satellites with the space environment, in order to develop mitigation means. Two projects, currently under negotiation, will start in 2014.

Another nine space weather related projects have been funded in various calls for proposals under the topic "Exploitation of space science and exploration data", which aims to add value to space missions and Earth-based observations by contributing to the effective scientific exploitation of collected data.

\section{Overview of the funded projects}

The purpose of this article is to provide a brief overview of the space weather related projects funded under the FP7 Space Theme.

As already mentioned, the funded research covers a wide range of solar physical phenomena, effects of space weather and applications. To provide an overview of the funded projects, they have been grouped in a simplified manner as shown in Figure 1. Although they focus on different phenomena, most of the projects funded under the topic "Exploitation of space science and exploration data" are grouped together as their goal is mainly to develop databases and models ensuring a better access to data.

Given the complexity and the wide scope of the topics researched by each project, only some examples of the main achievements are provided here, with references to websites and other documents for a more exhaustive view on the projects' outcomes. The different dissemination or confidentiality policies adopted by each project on their results, as well as

\footnotetext{
${ }^{6}$ Cooperation Work Programme 2010, Theme 9 - Space, European Commission C (2009) 5893 of 29 July 2009.
}

the extent of advancement in the activities, are also reflected in the level of detail provided for each project.

\subsection{Effects on space systems}

The SPACECAST project $^{7}$ is designed to develop European modelling and forecasting capabilities in order to help protect satellites on orbit from high-energy particles in the electron radiation belts and SEP events, two of the most important radiation hazards for space vehicles and manned spacecraft. Recent results were presented in the paper "Forecasting the Earth's radiation belts and modelling SEP events: Recent results from SPACECAST" by Horne et al. (2013).

The project developed the first European system providing a near-real-time (up to 3 hours ahead) forecast of the highenergy electron radiation belt (http://fp7-spacecast.eu/ index.php?page $=$ he forecasts). The forecasts are unique in that they use physical models, which include wave-particle interactions, and they cover the whole of the outer radiation belt comprising geostationary orbit, medium Earth orbit (where the European Galileo satellites orbit the Earth) and the slot region where new satellites are planned to operate. The forecast is stakeholder-driven and provides also a risk index for the satellite operations industry. In a second step of development the forecasting system was extended to provide a nowcast of the medium energy electrons (energies of 40-150 keV), which can cause surface charging on satellites and damage components including solar cells.

SPACECAST has also a strong focus on basic research. A database of different types of very low-frequency radio waves observed by seven different satellites has been developed and used to calculate new diffusion coefficients. The results integrated in the global radiation belt models contributed to improve the forecasts. Twelve extensive computer simulations of SEP events between 5.8 and 60 solar radii have enabled the development of a Coronal Shock Acceleration model. Based on diffusive shock acceleration theory, the model is now being used to develop a semi-analytical parameterisation for the foreshock region (just upstream of the shock) for a SEP prediction model.

Greater understanding of plasmaspheric dynamics and the influence of this upon the radiation belts is also the objective of the project PLASMON - A new, ground-based data assimilative model of the Earth's Plasmasphere - a critical contribution to Radiation Belt modeling for Space Weather purposes (http://plasmon.elte.hu). The project started by extending three ground-based measurement networks, namely AWDANet (VLF/whistlers), EMMA/SANSA (ULF/field line resonances), and AARDDVARK (VLF/perturbations on transmitters' signal) with new stations, and by improving the respective automatic detection and analysis algorithms.

The first results of the project are presented in the paper "The plasmasphere during a space weather event: first results from the PLASMON project" by Lichtenberger et al. (2013). Among these it is worth mentioning the development of a particle filter-based data assimilation model of the Earth's plasmasphere using ground-based plasma density observations as well as available satellite measurements as data input. Using the ground-based AARDDVARK network, the project developed also Relativistic Electron Precipitation models to monitor the

\footnotetext{
${ }^{7}$ SPACECAST - Protecting space assets from high-energy particles by developing European dynamic modelling and forecasting capabilities http://fp7-spacecast.eu/.
} 


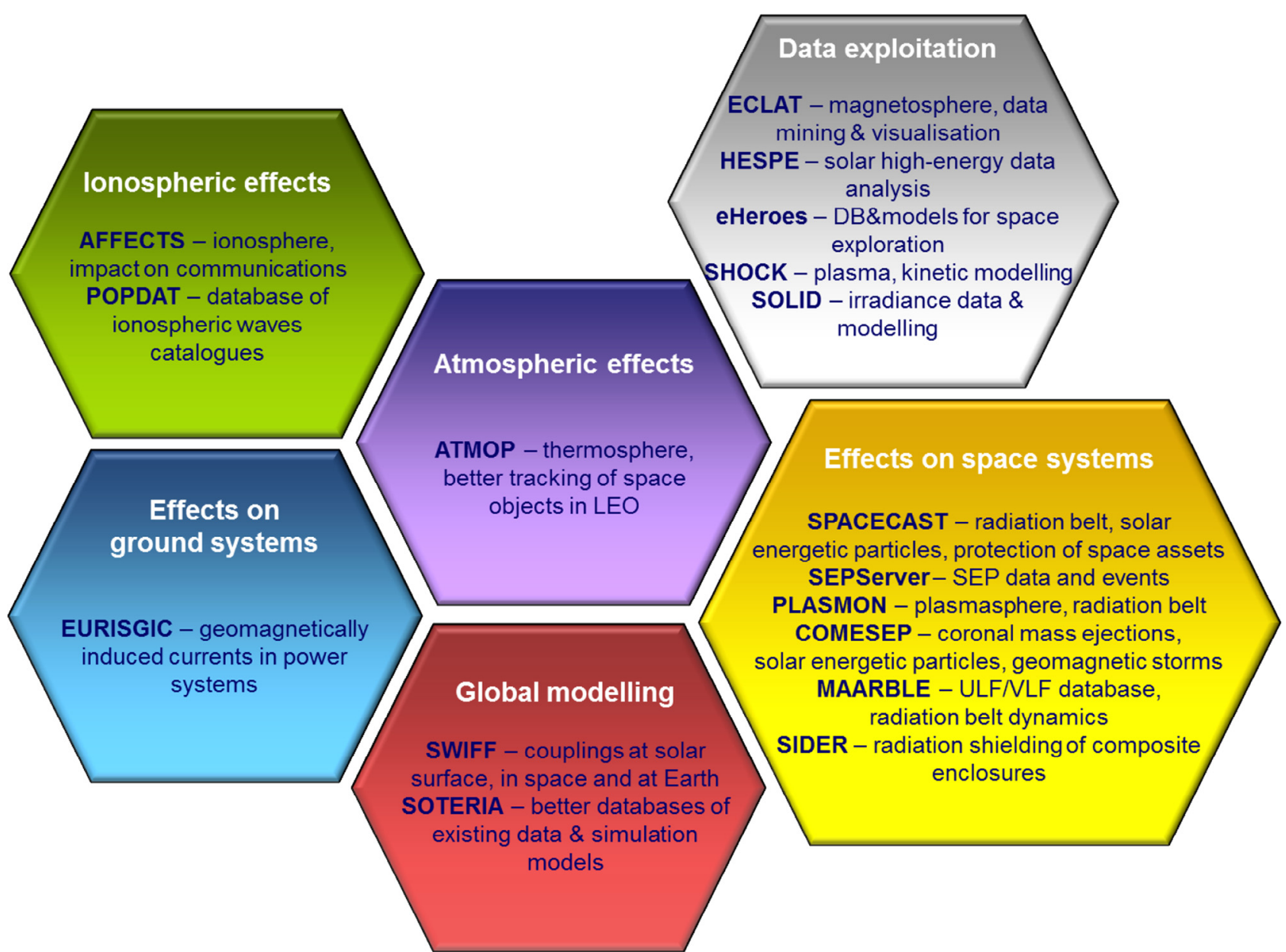

Figure 1. Space weather related projects funded under the FP7 Space Theme grouped according to the effects of space weather which they address.

losses of energetic particles and use them to compare the plasmasphere model dynamics.

PLASMON is a good example of the international cooperation encouraged by the Commission to address global challenges within certain areas of research. Given the nature of the system where any ground-based station samples one local time, the project requires data contributions from many local time sectors at the same time, and hence the participation of entities not only across Europe, but across the world. Together with European organisations, PLASMON involves partners from other space-faring nations, namely New Zealand, South Africa and the US, which contribute with their specific expertise or the utilisation of their specific geographic (geomagnetic) location(s).

Funded under the topic "Exploitation of space science and exploration data", the project MAARBLE - Monitoring, Analyzing and Assessing Radiation Belt Loss and Energization (www.maarble.eu) employs multi-spacecraft monitoring of the geospace environment, complemented by ground-based monitoring, in order to analyse and assess the physical mechanisms leading to radiation belt particle energisation and loss. Particular attention is paid to the role of ULF/VLF waves. A database containing properties of the waves is being created and will be made available to the scientific community. Based on the wave database, a statistical model of the wave activity dependent on the level of geomagnetic activity, solar wind forcing and magnetospheric region is being developed.
Also a "data exploitation" project, SEPServer - Data Services and Analysis Tools for Solar Energetic Particle Events and Related Electromagnetic Emissions (www.sepserver.eu) aims to provide access to state-of-the-art observations and analysis tools for the scientific community on SEP events and related electromagnetic (EM) emissions. SEPServer intends to lead to new knowledge on the production and transport of SEPs during solar eruptions and facilitate the development of models for predicting solar radiation storms and calculation of expected fluxes/fluences of SEPs encountered by spacecraft in the interplanetary medium.

The SEPServer catalogue of SEP events for the 23rd solar cycle, available online at http://server.sepserver.eu, consists of 115 proton events extending to energies $>55 \mathrm{MeV}$ observed by SOHO/ERNE over the years 1996-2010. The first results from the systematic analysis work performed on the datasets SOHO/ERNE, SOHO/EPHIN, ACE/EPAM, Wind/WAVES and GOES X-rays are presented in the paper "The first SEPServer event catalogue $\sim 68 \mathrm{-MeV}$ solar proton events observed at 1 AU in 1996-2010" by Vainio et al. (2013). In a companion paper "On the parametrization of the energetic-particle pitchangle diffusion coefficient" by Agueda \& Vainio (2013) the performance of different methods for pitch-angle scattering simulation is analysed.

Scientists from the solar energetic particle (SEP), coronal mass ejection (CME) and terrestrial effects scientific communities collaborate in the frame of the project 
COMESEP - COronal Mass Ejections and Solar Energetic Particles: forecasting the space weather impact (www.comesep.eu), to build an operational European Space Weather Alert system able to dispatch alerts and forecasts in a human-readable format on harmful space weather events in near-real time.

The project has developed tools for forecasting geomagnetic storms and SEP radiation storms, has examined and modelled the sources and propagation of SEPs, and has investigated the structure, propagation and evolution of CMEs to enhance the understanding of their 3D kinematics and interplanetary propagation. The developed tools have been validated and integrated in the prototype of the space weather alert system, which will be launched during the 10th European Space Weather Week meeting in November 2013. Geomagnetic and SEP radiation storm alerts are based on the COMESEP definition of risk.

In the project SIDER - Radiation Shielding of Composite Space Enclosures (www.sider-project.eu) a technology based on nanomaterials (doping of the bulk resin with nanofillers and infiltration of resin into buckypaper) is proposed as an alternative for the radiation shielding of sensitive satellite components. A second strategy based on the incorporation of a high density material foil is also being considered. The radiations test at two orbits, namely Low-Earth Orbit and Geostationary orbit, and the physical simulations of the space radiation interaction with satellite structure materials, electronics enclosures and device cases carried out during the second year of activity, demonstrated that the developed shielding solutions allow significantly reducing, without compromising safety, the weight of the structural elements.

\subsection{Ionospheric effects}

The project AFFECTS - Advanced Forecast For Ensuring Communications Through Space (www.affects-fp7.eu) aims to develop an advanced prototype space weather warning system to safeguard the operation of telecommunication and navigation systems on Earth from the threat of solar storms.

After two years of activity, an operational space weather early-warning system, with special emphasis on GNSS users and service operators, has been implemented on the German Aerospace Centre's SWACI ${ }^{8}$-AFFECTS website (http://swaciwebdevelop.dlr.de/early-warning-gnss/). The warning message, currently distributed to a small group of pilot users, will inform customers of space-based radio systems used in telecommunication, navigation/positioning and remote sensing of potential performance degradation of their systems due to space weather events at least one day in advance.

The individual forecasting models and tools developed in AFFECTS are being consolidated in the Forecast System Ionosphere (FSI), which includes short-term forecasting of geomagnetic indices, auroral alert and tracking system, as well as a software tool for forecasting perturbed Total Electron Content. To date the early-warning module delivers a warning mail with information on the status of the ionosphere to the test user group. FSI outputs for the already integrated modules can be found at the webpage http://swaciweb.dlr.de/AFFECTS/.

The Ionosphere Wave Service ${ }^{9}$ is a data mining service accessing a collection of topical catalogues, which characterise a number of Atmospheric Gravity Waves, Travelling

\footnotetext{
${ }^{8}$ Space Weather Application Center - Ionosphere (SWACI) http://swaciweb.dlr.de/.

9 Available at http://popdat.cbk.waw.pl.
}

Ionosphere Disturbances and Whistlers events. The service is the result of the project POPDAT-Problem-oriented Processing and Database Creation for Ionosphere Exploration (www.popdat.org), funded under the 2010 call topic "Exploitation of space science and exploration data" and concluded in April 2013. The project intended to add value to old ionosphere mission data and provide the scientific community with a new insight on wave processes taking place in the ionosphere.

\subsection{Atmospheric effects}

The Earth's thermosphere undergoes huge changes in response to solar and geomagnetic activity. Despite the number of efforts over the past couple of decades to physically and chemically model the upper atmosphere, there is a pressing need for a near-real-time thermosphere prediction model and operational services, which would enable the precise computation of air drag on low-Earth orbiting satellites. Orbit determination methods are necessary to predict accurately hours to days ahead the trajectory of space objects, which could pose a risk to space (due to possible collisions) or ground assets (due to re-entry objects).

The project ATMOP - Advanced Thermosphere Modelling for Orbit Prediction (www.atmop.eu) aims to develop a thermosphere model with operational nowcasting and forecasting capabilities, in order to perform reliable thermospheric air drag calculations, which can be applied to satellite orbit tracking, track loss, collisions and re-entry zone situations.

In the paper "Evaluation of the DTM-2009 thermosphere model for benchmarking purposes" (Bruinsma et al. 2012) the Drag Temperature Model (DTM2009 and DTM2000) and the COSPAR reference models NRLMSISE-00 and JB2008 are evaluated in order to establish benchmark values for new DTM models. Meanwhile the project released the first of two new semi-empirical thermospheric models in the autumn 2012 (http://www.atmop.eu/index.php/models). The new model DTM-2012 is fitted to an extended range of data, and comparison with current reference models indicates that it gives more accurate results in the $250-1000 \mathrm{~km}$ altitude range. DTM-2013 will be released by the end of the project in December 2013, taking into account $\mathrm{GOCE}^{10}$ data and new solar and geomagnetic proxies as input.

In parallel, work is on-going in the area of physical modelling of the thermosphere and developing schemes for near-realtime assimilation of thermospheric and ionospheric data into an advanced predictive DTM and into the physical Coupled Middle Atmosphere-Thermosphere (CMAT2) model.

From a strategic point of view, the project ATMOP could enable Europe to take a strategically leading position in the field of satellite drag modelling by making the Drag Temperature Model a reference in this field.

\subsection{Effects on ground systems}

The impact of Geomagnetically Induced Currents (GIC) on the electrical power networks in Europe, potentially resulting in a damage of the power transmission infrastructure and a blackout, is one of the key risks for the European citizens from a major solar event. The risk assessment, the production of worst case scenarios and the demonstration of the GIC forecasting capability, proposed by the project EURISGIC - European Risk from

\footnotetext{
${ }^{10}$ Gravity Field and Steady-State Ocean Circulation Explorer (GOCE).
} 
Geomagnetically Induced Currents (http://www.eurisgic.eu/), will help European stakeholders to consider the risks and decide on mitigation methods.

EURISGIC proposes the first assessment of the GIC risk at the European level. The project aims to produce the first European-wide prototype forecast tool based on in situ solar wind observations and on magnetosphere-ionosphere simulations to produce real-time GIC warnings for the European high-voltage power grids. While the development of the forecast service of GIC in Europe is still on-going, in the first two years of activity the project provided a complete set of tools to model GIC in continental-scale power grids. The paper "Continental scale modelling of geomagnetically induced currents" by Viljanen et al. (2012) presents the new features of the methodology utilised by the project to update GIC modelling, previously applied to national power grids in small geographical areas, to be able to cover the whole European region.

In addition to using readily available sources of data, the EURISGIC project is running GIC measurements at several sites in Finland, the UK, Hungary and North-West Russia, while historic geomagnetic and geoelectric analogue data are being digitised.

By utilising geomagnetic recordings and a developed prototype grid model, EURISGIC derived a geographic map which indicates the statistical occurrence of rapid geomagnetic variations and large geoelectric fields throughout Europe, as well as of large GIC in the prototype high-voltage power grid model (http://riskmap.eurisgic.eu/).

\subsection{Global modelling}

Concluded in 2011, SOTERIA - SOlar-TERrestrial Investigations and Archives (http://soteria-space.eu/) contributed to make better use of existing data and developed databases, going beyond the present state-of-the-art in regard to details, time-resolution and improved methods of accessing data. The studies conducted by SOTERIA involved the analysis and processing of the relevant data from 18 satellites, including several ESA and other European satellites, complemented by a large set of data from European ground-based observatories. SOTERIA deployed also a considerable effort in utilising and developing theoretical and simulation models for interpreting the space weather data, covering all aspects of the complex Sun-Earth connection. SOTERIA also provided guidance for future investigations as manifested in the article "New observation strategies for the solar UV spectral irradiance" by Cessateur et al. (2012).

The large inventory of results, deliverables and reports are publicly available via the SOTERIA website and the European Space Weather Portal (http://www.spaceweather.eu/fr/repository). A web interface with all the predictive tools developed by SOTERIA is available for the public to predict future space weather (http://soteria-space.eu/spacetools.php). One of the main achievements is the SOTERIA Virtual Observatory (http://soteria.oma.be:8080/soda/query.iface) gathering all the observational data collected by SOTERIA and providing it in a rationalised and organised way via a web interface based on state-of-the-art software.

The project SWIFF - Space Weather Integrated Forecasting Framework (http://swiff.eu/) addresses two fundamental issues in space weather: the development of an integrated framework for the mathematical-physics models which form the basis for space weather forecasting, and the study of methods and software to address the linkage (coupling) between different physics and processes developing simultaneously or in cascade at the Solar Corona, in the interplanetary space and in the interaction at the Earth magnetosphere. The project plan starts from the fundamental physics of the space weather processes and designs mathematical models best suited to accurately represent such processes, proceeds to develop computational algorithms best suited to the models at hand and finally implements a common integrated software infrastructure to make space weather forecasting possible.

In the first two years of activity SWIFF has collected some of the best and most promising techniques to handle multiphysics and multiscale problems and has demonstrated the possibility of these methods in selected processes relevant to space weather modelling, most notably the processes of energy exchange between magnetic fields and plasmas. The developed tools have been applied to solar corona problems, to the interaction of the interplanetary space and its perturbations with the Earth magnetosphere and to the inner magnetospheric environment, see Lapenta et al. (2013) for selected results. A very first fully kinetic model of the Earth environment, including the plasmasphere and radiation belts within a single first principle kinetic treatment of the whole magnetosphere, and a fully integrated model of coronal evolution and particle acceleration are examples of the project results.

\subsection{Exploitation of space science and exploration data}

The project ECLAT - European Cluster Assimilation Technol$o g y^{11}$ will deliver a novel database and tools for space scientists, by providing an upgrade of the European Space Agency's Cluster Active Archive (CAA), which is a state-ofthe-art space plasma physics data repository containing over 10 years of magnetospheric observations from the ESA Cluster multi-spacecraft mission. ECLAT will ingest into the CAA supporting data from other space- and ground-based observatories, provide data mining routines, refine data products and software tools for their visualisation, and develop existing European magnetospheric modelling infrastructure to provide context for the observational data.

The achievements of the project to date include, for instance, the production of Cluster Exchange Format files for the Cluster C3 ionospheric footprints at $120 \mathrm{~km}$ altitude for each day of the period 2001-2009 and the corresponding ionospheric footprint quick-look plots; as well as observations of the global ionospheric convection pattern for the years 20002010, determined by the Super Dual Auroral Radar Network (SuperDARN) and the production of the corresponding hourly, daily and monthly quick-look plots for rapid browsing of the SuperDARN data and event-selection.

The rationale of the project HESPE - High Energy Solar Physics Data in Europe (www.hespe.eu) is to formulate and implement computational methods for solar high-energy data analysis, and to utilise Information and Communication Technology tools for providing algorithms and science-ready products to the solar physics community.

One of the main achievements to date has been the development of a distributed and autonomous HESPE processing framework, which allows for autonomous and distributed processing of flare data, collected by either the Reuven Ramaty High Energy Solar Spectroscopic Imager (RHESSI) or any other instrument for high-energy measurements based on modulation profiles. It manages all the necessary steps from reading

\footnotetext{
${ }^{11} \mathrm{http}: / / w w w . s p a c e . i r f u . s e / E C L A T / e c l a t-w e b / e c l a t$ detail.html.
} 
raw data and flare-specific configurations, to processing the data and publishing the visibility database and science products.

eHeroes - Environment for Human Exploration and RObotic Experimentation in Space (www.eheroes.eu) adopts a synergistic approach using models, simulations and observational data to characterise the environment in space to provide useful information for evaluating the impact on human and robotic space exploration. The project aims at realising effective scientific exploitation of space data by combining different datasets from current robotic explorations of the Solar System with data from current and past space-based and complementing ground-based observations, together with new data sources from the latest missions. eHEROES adds value to the data by better organising and cross-linking them, by applying state-ofthe-art models and by assimilating the heliospheric data in space environment simulations.

The project has a space climate component aiming at predicting the best periods in the solar cycle to complete space missions, and characterising the different threats in the different periods. The resulting information and services should be useful to plan and operate future space missions to the Moon and Mars as well as in the near-Earth environment.

Taking advantage of the synergies between space plasma modelling and data analysis, the project SHOCK - Solar and Heliospheric Collisionless Kinetics ${ }^{12}$ aims to advance the understanding of the Sun to Earth plasma system and fundamental processes such as turbulence, shocks, particle acceleration and reconnection. It will increase the use of modelling and kinetic simulation in the analysis of space data from the European Space Agency's missions such as Cluster, Cassini, Ulysses, Helios, BepiColombo and Solar Orbiter, but also from international missions such as Themis, MMS, Solar Probe Plus, etc. and ground-based observatories.

As a key outcome to date, the project has developed a prototype of the Virtual Mission Laboratory, demonstrating a webbased tool for visualising simulation data in a format for comparison with spacecraft mission data (http://amalka.asu.cas.cz/ esa/).

The project SOLID - First European Comprehensive SOLar Irradiance Data exploitation (http://projects. pmodwrc.ch/solid/) focuses on the comprehensive analysis of solar irradiance variations, which are the most important natural factor driving the terrestrial climate and as such a crucial input to any climate modelling. Started in 2013, the project will combine a large variety of different solar spectral irradiance data sets (spectral measurements, solar images and solar proxies) to deliver a single continuous and high level solar spectral irradiance record, which covers the space era from 1979 onwards. Two different state-ofthe art approaches are adopted to reconstruct spectral and total solar irradiance data as a function of time. These results will be used to bridge gaps in time and wavelength coverage of the observational data, which will allow to reduce the uncertainties in the irradiance time series - an important requirement by the climate community - and to provide uniform data sets of modelled and observed solar irradiance data from the beginning of the space era to the present including proper error and uncertainty estimates.

\section{Space weather research in Horizon 2020}

Space research is supported in the incoming EU Framework Programme for Research and Innovation - Horizon 2020 which will run from 2014 to $2020^{13}$, under the heading "Industrial Leadership", in line with the main objective and challenge to foster a cost-effective competitive and innovative space industry (including SMEs) and research community to develop and exploit space infrastructure to meet future Union policy and societal needs. Building on the successes of FP7, Horizon 2020 will enable the European space research community to develop innovative space technologies and operational concepts "from idea to demonstration in space", and to use space data for scientific, public or commercial purposes.

In the Space Work Programme 2014-2015 approximately $60 \%$ of the budget should be allocated to the three EU Space flagships initiatives: European Global Navigation Satellite System (EGNSS), Earth Observation and Protection of European assets in and from space.

Although great progress has been made in comprehending the solar processes, further research is still necessary to understand the physical phenomena at the origin of space weather including the Sun-Earth interaction chain and to convert the scientific knowledge into an operational service. Taking into account the results of the FP7-funded projects and the new services being developed, Horizon 2020 calls for proposals on Space Weather ${ }^{14}$ would address issues like exploratory work on new ideas for analysing the expected larger volumes of data from existing and upcoming missions. Improved understanding and more accurate modelling of the complex and interacting physical phenomena would be expected to enable improved forecasting of space weather events affecting the Earth and the near-Earth space environment.

\section{References}

Agueda, N., and R. Vainio, On the parametrization of the energeticparticle pitch-angle diffusion coefficient, J. Space Weather Space Clim., 3, A10, DOI: 10.1051/swsc/2013034, 2013.

Bruinsma, S., N. Sánchez-Ortiz, E. Olmedo, and N. Guijarro, Evaluation of the DTM-2009 thermosphere model for benchmarking purposes, J. Space Weather Space Clim., 2, A04, DOI: 10.1051/swsc/2012005, 2012.

Cessateur, G., J. Lilensten, T. Dudok de Wit, A. BenMoussa, and M. Kretzschmar, New observation strategies for the solar UV spectral irradiance, J. Space Weather Space Clim., 2, A16, DOI: 10.1051/swsc/2012016, 2012.

Horne, R., S. Glauert, N. Meredith, H. Koskinen, R. Vainio, et al., Forecasting the Earth's radiation belts and modelling solar energetic particle events: Recent results from SPACECAST, $J$. Space Weather Space Clim., 3, A20, DOI: 10.1051/swsc/2013042, 2013.

Lapenta, G., V. Pierrard, R. Keppens, S. Markidis, S. Poedts, et al., SWIFF: Space weather integrated forecasting framework, J. Space Weather Space Clim., 3, A05, DOI: 10.1051/swsc/2013027, 2013.

Lichtenberger, J., M. Clilverd, B. Heilig, M. Vellante, J. Manninen, et al., The plasmasphere during a space weather event: first results from the PLASMON project, J. Space Weather Space Clim., 3, A23, DOI: 10.1051/swsc/2013045, 2013.

\footnotetext{
12 SHOCK - Solar and Heliospheric Collisionless Kinetics: Enabling Data Analysis of the Sun to Earth Plasma System with Kinetic Modelling - http://project-shock.eu/.
} 
P. Chiarini: Space Weather in the EU's FP7 space theme

Vainio, R., E. Valtonen, B. Heber, O. Malandraki, A. Papaioannou, et al., The first SEPServer event catalogue $\sim 68 \mathrm{-MeV}$ solar proton events observed at 1 AU in 1996-2010, J. Space Weather Space Clim., 3, A12, DOI: 10.1051/swsc/2013030, 2013.
Viljanen, A., R. Pirjola, M. Wik, A. Ádám, E. Prácser, et al., Continental scale modelling of geomagnetically induced currents, J. Space Weather Space Clim., 2, A17, DOI: 10.1051/swsc/2012017, 2012.

Cite this article as: Chiarini P: Space weather in the EU's FP7 Space Theme. J. Space Weather Space Clim., 2013,3 , E01. 